\title{
Repeated touch and needle-prick stimulation in the neonatal period increases the baseline mechanical sensitivity and postinjury hypersensitivity of adult spinal sensory neurons
}

Nynke J. van den Hoogen ${ }^{\mathrm{a}, \mathrm{b}}$, Jacob Patijn ${ }^{\mathrm{a}}$, Dick Tibboel ${ }^{\mathrm{c}}$, Bert A. Joosten ${ }^{\mathrm{a}, \mathrm{b}}$, Maria Fitzgerald ${ }^{\mathrm{d}}$, Charlie H.T. Kwok ${ }^{\mathrm{d}, *}$

\begin{abstract}
Noxious stimulation at critical stages of development has long-term consequences on somatosensory processing in later life, but it is not known whether this developmental plasticity is restricted to nociceptive pathways. Here, we investigate the effect of repeated neonatal noxious or innocuous hind paw stimulation on adult spinal dorsal horn cutaneous mechanical sensitivity. Neonatal Sprague-Dawley rats of both sexes received 4 unilateral left hind paw needle pricks (NPs, $n=13$ ) or 4 tactile (cotton swab touch) stimuli, per day (TC, $n=11$ ) for the first 7 days of life. Control pups were left undisturbed ( $n=17)$. When adult (6-8 weeks), lumbar wide-dynamic-range neuron activity in laminae III-V was recorded using in vivo extracellular single-unit electrophysiology. Spike activity evoked by cutaneous dynamic tactile (brush), pinch and punctate (von Frey hair) stimulation, and plantar receptive field areas were recorded, at baseline and 2 and 5 days after left plantar hind paw incision. Baseline brush receptive fields, von Frey hair, and pinch sensitivity were significantly enhanced in adult NP and TC animals compared with undisturbed controls, although effects were greatest in NP rats. After incision, injury sensitivity of adult wide-dynamic-range neurons to both noxious and dynamic tactile hypersensitivity was significantly greater in NP animals compared with TC and undisturbed controls. We conclude that both repeated touch and needle-prick stimulation in the neonatal period can alter adult spinal sensory neuron sensitivity to both innocuous and noxious mechanical stimulation. Thus, spinal sensory circuits underlying touch and pain processing are shaped by a range of early-life somatosensory experiences.
\end{abstract}

Keywords: NICU, Neonatal sensory intervention, Tactile stimulation, Early-life pain, Postoperative

\section{Introduction}

Chronic pain affects the daily lives of 1 in 5 adults worldwide and remains undertreated. ${ }^{14}$ Successful therapeutic solutions for pain are hampered by the diversity in chronic pain mechanisms and individual differences in pain sensitivity. ${ }^{45}$ Recent research suggests that early-life exposure to abnormal sensory stimulation is a major determinant for future pain susceptibility. Neonates

$\overline{\text { Sponsorships or competing interests that may be relevant to content are disclosed }}$ at the end of this article.

${ }^{a}$ Department of Anaesthesiology and Pain Management, Maastricht University Medical Centre+, Maastricht, the Netherlands, ${ }^{b}$ Department of Translational Neuroscience, School of Mental Health and Neuroscience, Maastricht University, Maastricht, the Netherlands, ${ }^{\circ}$ Intensive Care, Department of Paediatric Surgery, Erasmus MC-Sophia, Rotterdam, the Netherlands, ${ }^{d}$ Department of Neuroscience, Physiology and Pharmacology, University College London, London, United Kingdom. Kwok is now with the Hotchkiss Brain Institute, University of Calgary, Calgary, Alberta, Canada.

${ }^{*}$ Corresponding author. Address: University of Calgary, HSC 2044, Calgary, Alberta, Canada T2N 4N1. Tel.: (+1) 403-210-7365. E-mail address: hongting.kwok@ ucalgary.ca (C.H.T. Kwok).

PAIN 159 (2018) 1166-1175

Copyright $\odot 2018$ The Author(s). Published by Wolters Kluwer Health, Inc. on behalf of the International Association for the Study of Pain. This is an open access article distributed under the Creative Commons Attribution License 4.0 (CCBY), which permits unrestricted use, distribution, and reproduction in any medium, provided the original work is properly cited.

http://dx.doi.org/10.1097/j.pain.00000000000001201 admitted to Neonatal Intensive Care Unit (NICU) typically receive 10 to 14 painful procedures and considerable handling each day, often without adequate preemptive analgesic. ${ }^{31}$ Several clinical studies have shown that such neonatal sensory interventions can lead to alterations in behavioural and neurophysiological measures of pain processing. ${ }^{8,15,25,38,40,41}$ Moreover, the analgesic requirement for subsequent surgery is higher in infants who have undergone surgical procedures during the neonatal period. ${ }^{27}$

The long-term effects of early-life injury on adult pain behaviour have been directly demonstrated in rodent laboratory models. ${ }^{36}$ In rodents, repeated neonatal needle pricks (NPs) lead to increased thermal and mechanical behavioural hypersensitivity in adulthood ${ }^{2,20}$ and hind paw incision in early life has been shown to increase reflex pain hypersensitivity to repeat surgical injury in adults. ${ }^{49}$ Other studies have also reported a decrease in thermal sensitivity after early-life surgeries ${ }^{39}$ and altered descending pain modulation after hind paw incision during the first week of life. ${ }^{47}$ Importantly, the immature central nervous system is highly plastic, and sensory activity in the first week of life can induce adaptive changes within the spinal dorsal horn. ${ }^{36,43}$ Thus, early-life NPs increase C-fibre terminal density, ${ }^{20}$ and early skin incision alters the spike timing dependence and local inhibitory processing in the adult spinal dorsal horn. ${ }^{23,24}$ These events can amplify nociceptive signalling in later life and result in hypersensitive nociceptive circuits, maintained by aberrant neuroimmune interactions. ${ }^{5,35}$ 
However, the modality selectivity of these changes is not clear. Other nonnoxious tactile sensory circuits may also be altered in these models, and indeed, similar plasticity may be induced in spinal sensory circuits after repeated neonatal tactile interventions as well as noxious procedures. Neonates in intensive care undergo repeated handling and tactile stimulation ${ }^{13,17}$ which may itself contribute to alterations in developing circuitry through activity-dependent mechanisms. ${ }^{21}$ This question can be studied most effectively at the level of dorsal horn circuits, where both innocuous and noxious sensory inputs are first integrated in the central nervous system. To date, there has been no study of the effect of repeated neonatal noxious or tactile procedures on neuronal excitability in the adult spinal dorsal horn.

This study investigates the effect of neonatal sensory interventions on the innocuous and noxious mechanical cutaneous sensitivity of adult dorsal horn neurons using in vivo electrophysiology. The neonatal interventions used were repetitive needle pricking and a matched repetitive, nonnoxious tactile stimulation using a cotton swab, compared with undisturbed controls. Because neonatal needle pricking is known to increase adult postinjury behavioural hypersensitivity, ${ }^{20,44}$ we also studied the postinjury tactile and nociceptive sensitivity of adult dorsal horn neurons after the same neonatal interventions.

\section{Methods}

\subsection{Animals}

All experiments were performed in accordance with the UK Animal (Scientific Procedures) Act 1986. Adult and neonatal male and female Sprague-Dawley rats were obtained from the Biological Services Unit, University College London. Animals were housed in a temperature and humidity controlled room with a 12:12-hour light/dark cycle with food and water available ad libitum. Rats were exposed to the same caging, diet, and handling throughout the experiment. Litters were weaned at postnatal day 21 (P21) and animals were housed in same sex cages of 3 to 4 littermates. Reporting is according to the Animal Research: Reporting of in vivo Experiments guidelines developed by the National Centre for Replacement, Refinement, and Reduction of Animals in Research, United Kingdom.

\subsection{Study design}

To assess the effect of repetitive sensory stimulations during the first week of life on the electrophysiological properties of spinal cord neurons in adulthood, repetitive neonatal NPs were applied to the left hind paw during the first postnatal week as described elsewhere. ${ }^{19,20,44}$ To control for neonatal stress and handling, age-matched littermates received tactile stimulation (with a cotton swab) on the plantar surface of the left hind paw. A separate group of animals from different litters was left undisturbed. After the first week, litters were allowed to grow into adulthood undisturbed. When adult, (postnatal weeks 6-8) a subset of the animals were anaesthetised and an incision applied to the left hind paw, which is a model of surgical/postoperative pain. In vivo extracellular single-unit recordings of spinal dorsal horn neurons were performed at baseline (before hind paw incision), and 1 to 2 and 5 to 6 days after incision. Table 1 provides an overview of the animals used in the study.

\subsection{Neonatal procedures}

New-born rat pups $(n=13)$ from 2 litters underwent 4 NPs per day from day of birth, postnatal day 0 (P0), to $\mathrm{P} 7$ as previously described. ${ }^{20}$ Each NP consisted of a single insertion of a sterile 25-G needle, $2 \mathrm{~mm}$ deep into the midplantar surface of the left hind paw. Age-matched littermates were used for the tactile stimulation (TC) group $(n=11)$, receiving 4 swabs with a cotton swab on the midplantar surface of the left hind paw per day, from P0 to P7. A separate group of animals from different litters was left undisturbed (UC, $n=17$ ). Mechanical sensitivity to von Frey hair (vFh) stimulation was tested daily during the first postnatal week before (baseline), 1, 3, and 5 hours after NP application, to assess the development of hypersensitivity in the foot. Briefly, pups were lightly restrained on a bench surface, and vFhs (in grams, 0.4, 0.6, 1.0, 2.0, 4.0 [from P4 on], and 6.0 [from P6 on]) were applied 5 times to the dorsal surface of the paw, for 1 second per application. The number of positive responses (ie, withdrawal or flinching responses evoked by vFhs, guarding of the foot) was recorded and a 50\% paw withdrawal threshold was calculated using sigmoidal curve fitting in GraphPad Prism 7.

\subsection{Adult skin incision}

A unilateral plantar hind paw incision was made as previously described. ${ }^{7}$ Briefly, at 6 to 8 weeks of age, animals were anaesthetised with isoflurane (2\%-3\%), an incision (1 cm long) was made along the midline through the skin and fascia of the left plantar hind paw, the plantaris muscle was then lifted and incised longitudinally. The skin was sutured with 2 mattress sutures (5-0 silk suture, Ethicon).

\subsection{Dorsal horn electrophysiology}

In vivo extracellular single-unit recordings were performed as previously described. ${ }^{37}$ Rats were anaesthetised with isoflurane (induction 5\% in $\mathrm{O}_{2}$ ). The animals were tracheotomised; air flow and breathing rate were adjusted to animal weight with a small animal ventilator (Model 687; Harvard Apparatus, MA). Procedures were performed under constant isoflurane anaesthesia (maintenance $1.8 \%$ in $\mathrm{O}_{2}$, Univentor Anaesthesia Unit 400; Royem Scientific, Luton, United Kingdom). A heating blanket with feedback control was used to maintain body temperature throughout recording (Model 507220F; Harvard Apparatus). The rat was mounted on a stereotactic frame (Kopf Instruments, Tujunga, CA) and a laminectomy performed to expose the lumbar spinal cord. The vertebral column was secured with a clamp rostral to the laminectomized area, the dura and pia mater were removed, and the exposed spinal cord was covered with mineral oil.

Table 1

Number of animals (male and female) used in the study.

\begin{tabular}{llll}
\hline Adult rats & Baseline & 2 days after skin incision & 5 days after skin incision \\
\hline Neonatal undisturbed (UC) & $7(6,1)$ & $5(5)$ & $5(5)$ \\
\hline Neonatal needle prick (NP) & $4(3,1)$ & $4(3,1)$ & $5(4,1)$ \\
\hline Neonatal tactile (TC) & $4(3,1)$ & $3(3)$ & $4(3,1)$ \\
\hline
\end{tabular}


Table 2

Number of wide-dynamic-range cells (ipsilateral and contralateral) recorded in each group.

\begin{tabular}{llll}
\hline Adult rats & Baseline & 2 days postoperative & $\mathbf{5}$ days postoperative \\
\hline Neonatal undisturbed (UC) $\mathrm{N}=17$ rats & $29(23,6)$ & $34(26,8)$ & $39(25,14)$ \\
\hline Neonatal needle prick (NP) $\mathrm{N}=13$ rats & $24(16,8)$ & $32(21,11)$ & $45(26,19)$ \\
\hline Neonatal tactile (TC) $\mathrm{N}=11$ rats & $34(22,12)$ & $30(16,14)$ & $31(16,15)$ \\
\hline
\end{tabular}

A 6- $\mu \mathrm{m}$ tipped glass-coated carbon fibre electrode (Kation Scientific, Minneapolis, MN) was lowered through the spinal cord in 2 to $10 \mu \mathrm{m}$ steps with a Microdrive (Scientifica Microdrive, Scientifica, Uckfield, United Kingdom). To isolate individual cells, the plantar surface of the hind paw was stroked as search stimulus for dorsal horn wide-dynamic-range (WDR) neurons in laminae $\mathrm{N}-\mathrm{VI}$, at a depth between 400 and $800 \mu \mathrm{m}$. Stimulus-evoked potentials were digitalized by a Powerlab interface and recorded using Labchart software (AD Instruments Ltd, Oxford, United Kingdom). The cutaneous receptive field (RF) on the plantar skin to tactile (a camel hair brush) and noxious pinch stimulation (fine forceps) was mapped using InkScape software. Spontaneous activity in the absence of cutaneous stimulation was recorded for 1 minute. The number of spikes evoked during a single 0.5-second brush (repeated 3 times at $>1$ second intervals), a single 1.5-second pinch (repeated 3 times at $>1$ second intervals), and a single $0.5-$ second vFh stimulus (range: 1.202; 2.041; 3.63; 5.495; 8.511; 15.136; and $28.84 \mathrm{~g} ; 3$ applications to peak RF, $>1$ second interstimulus interval) was recorded, with a minimum 10-second interval between stimuli. A total of 313 cells were recorded from the ipsilateral and contralateral dorsal horn of 41 animals (206 ipsilateral and 107 contralateral). Outliers were removed and 298 cells (191 ipsilateral and 107 contralateral) were included in the analysis, summarized in Table 2. The depth of cells recorded and analysed per group is included in Table 3.

\subsection{Statistical analysis}

All data were plotted using GraphPad Prism 7 and presented as mean \pm SEM. A $P$ value $<0.05$ was considered statistically significant. Statistical outliers were identified by the ROUT's method in GraphPad Prism, 15 of 313 cells recorded were excluded from analysis as outliers. Normality of data sets was checked using the D'agostino and Pearson test. As data sets were normally distributed, parametric tests were applied.

\section{Table 3}

Depth of cells ( $\mu \mathrm{m}$, wide-dynamic-range neurons) recorded in the spinal dorsal horn of each group.

\begin{tabular}{lcr}
\hline & \multicolumn{1}{c}{ Ipsilateral } & \multicolumn{1}{c}{ Contralateral } \\
\hline Baseline & & \\
Neonatal needle prick (NP) & $547.21 \pm 36.1$ & $523.29 \pm 31.36$ \\
Neonatal tactile (TC) & $653.37 \pm 15.82$ & $592.14 \pm 25.45$ \\
Neonatal undisturbed (UC) & $533.11 \pm 24.66$ & $502.97 \pm 40.98$ \\
\hline 2 days & & \\
Neonatal needle prick (NP) & $493.5 \pm 22$ & $579.93 \pm 38.48$ \\
Neonatal tactile (TC) & $608 \pm 17.14$ & $603.79 \pm 19.03$ \\
Neonatal undisturbed (UC) & $531.08 \pm 21.56$ & $541.56 \pm 40.17$ \\
\hline 5 days & & \\
Neonatal needle prick (NP) & $595.42 \pm 22.89$ & $548.57 \pm 30.05$ \\
Neonatal tactile (TC) & $575.07 \pm 26.9$ & $606 \pm 28.45$ \\
Neonatal undisturbed (UC) & $544.8 \pm 18.67$ & $582.15 \pm 28.44$ \\
\hline
\end{tabular}

Neonatal behavioural data were analysed using 2-way analysis of variance (ANOVA) with post hoc Bonferroni multiple comparison. Group differences in neuronal activity at baseline (before incision, at 6-8 weeks of age) were compared using 1-way ANOVA with post hoc Bonferroni multiple comparison. Withinand between-group differences in neuronal activity after incision were compared with the 2-way ANOVA test with post hoc Bonferroni (postincision) multiple comparison.

\section{Results}

\subsection{Repetitive neonatal needle prick, but not tactile stimulation, transiently decreases mechanical withdrawal thresholds}

Needle pricks or tactile (cotton swab touch) stimulation (TC) was applied 4 times a day to the left (ipsilateral) hind paw from postnatal day (P)0-P7 ( $\mathrm{N}=19$ male, 5 female). Behavioural mechanical reflex withdrawal thresholds were tested with vFhs before and after each stimulation session at 1, 3, and 5 hours (Fig. 1A), in both the ipsilateral and contralateral hind paws. Figure 1B shows that the thresholds in the ipsilateral hind paws of TC animals are unaffected by repeated tactile stimulation, displaying the normal developmental increase in reflex withdrawal thresholds reported for naive animals. ${ }^{18} \mathrm{~A}$ fall in threshold relative to the TC group was observed after NPs in all time points from P3-7 in the ipsilateral hind paws (Fig. 1B; TC vs NP at P3: +1 : $P<$ $0.001,+3 P<0.0001,+5 P<0.01 ; P 4:+1 P<0.0001,+3$ and $+5 P<0.001$; P5: $+1 P<0.05,+3$ and $+5 P<0.01 ; P 6:+1$ $P<0.001,+3 P<0.01,+5 P<0.0001 ; \mathrm{P} 7:+1 P<0.0001,+3$ $P<0.001,+5 P<0.0001$, 2-way ANOVA with post hoc Bonferroni). Baseline NP mechanical withdrawal thresholds recovered each day, but by the end of the week, they were significantly lower than TC baseline thresholds (P6 NP $0.95 \pm$ $0.11 \mathrm{~g}$ vs TC $1.34 \pm 0.11 \mathrm{~g}, P<0.05$, 2-way ANOVA with post hoc Bonferroni).

In addition, in the contralateral hind paws of NP animals, a fall in threshold relative to TC group was observed at 1 hour after NPs at P6, and at baseline and 5 hours after NPs at P7 (Fig. 1C; P6+1 $\mathrm{NP} 0.79 \pm 0.25 \mathrm{~g}$ vs TC $1.4 \pm 0.38 \mathrm{~g}, P<0.0001 ; \mathrm{P} 7$ BS NP $1.32 \pm 0.46 \mathrm{~g}$ vs $1.9 \pm 0.32 \mathrm{~g}, P<0.0001 ; \mathrm{P} 7+5 \mathrm{NP} 1.51 \pm 0.53$ $\mathrm{g}$ vs $1.95 \pm 0.34 \mathrm{~g}, P<0.01,2$-way ANOVA with post hoc Bonferroni).

\subsection{Baseline sensitivity to cutaneous brush and noxious pinch stimulation is increased in adult dorsal horn neurons after early-life touch and needle prick}

The rats were left to grow up to 6 to 8 weeks old and the properties of adult dorsal horn WDR sensory neurons were investigated using in vivo extracellular single-unit electrophysiology (Fig. 2A). Neurons with RFs on the plantar surface of the ipsilateral and contralateral hind paw were recorded from the L4/5 dorsal horn in NP, TC, and naive (neonatally undisturbed) 
adult rats. Although ipsilateral measurements provide information on sensory processing in the neonatally stimulated dermatome, contralateral measurements indicate global changes in cutaneous mechanosensitivity. Baseline sensitivity of WDR cells to dynamic tactile (brush) stimulation of the plantar skin, measured as mean spikes per stimulus and mean RF area, is shown in Figures 2B-D. Baseline sensitivity to noxious mechanical (pinch) stimulation, using the same measures, is shown in Figures $2 \mathrm{E}$ and $\mathrm{F}$.

Mean spikes per stimulus to brush was significantly greater in NP animals compared with naive animals at baseline (Fig. 2B; $F$ $(2,61)=6.671, P<0.01$, naive vs NP: $7.1 \pm 0.71$ vs $14.45 \pm$ 1.92; $P<0.01$, 1-way ANOVA with post hoc Bonferroni), but not in TC animals. Contralateral cells were unaffected. Ipsilateral brush RF area at baseline was significantly larger in both TC and $\mathrm{NP}$ animals compared with naive controls (Fig. 2C; $\mathrm{F}(2,60)=$ 41.71, $P<0.0001$, 1-way ANOVA), with mean NP RFs being significantly larger than TC (naive vs TC: $2614 \pm 275.9$ vs $4767 \pm$ 394.7, $P<0.01$; naive vs NP $2614 \pm 275.9$ vs $8590 \pm 724.2, P<$ 0.0001 ; TC vs NP $4767 \pm 394.7$ vs $8590 \pm 724.2, P<0.0001$; 1-way ANOVA with post hoc Bonferroni). The mean brush RF area in the contralateral paw of NP animals was also significantly greater compared with naive and TC animals (Fig. 2D; naive vs NP $1175 \pm 177.6$ vs $8837 \pm 1690, P<0.0001$; TC vs NP $2511 \pm$
369.6 vs $8837 \pm 1690, P<0.05 ; 1$-way ANOVA with post hoc Bonferroni).

Mean spikes per pinch stimulus were also significantly greater in TC and NP animals than in naive animals at baseline (Fig. 2E; F $(2,63)=4.96, P<0.01$; naive vs TC $68.08 \pm 8.72$ vs $105.4 \pm$ 10.73, $P<0.05$; naive vs NP $68.08 \pm 8.72$ vs $111.8 \pm 14.2, P<$ 0.05 ; 1-way ANOVA with post hoc Bonferroni). In addition, mean ipsilateral pinch RF area was significantly larger in both TC and NP animals compared with naive controls and was largest in NP animals (Fig. 2F; $F(2,61)=38.23, P<0.0001$, naive vs TC 742.1 \pm 97.6 vs $1991 \pm 170.9, P<0.0001$; naive vs NP $742.1 \pm 97.6$ vs $2933 \pm 267.5, P<0.0001$; TC vs NP $1992 \pm 170.9$ vs $2933 \pm$ 267.5, $P<0.01$; 1-way ANOVA with post hoc Bonferroni).

\subsection{Postinjury sensitivity to cutaneous dynamic tactile and noxious pinch stimulation is increased in adult dorsal horn neurons after early-life touch and needle prick}

The results above showed significant increases in adult dynamic tactile and noxious mechanical sensitivity in NP and TC dorsal horn cells measured at baseline. We next asked whether postinjury sensitivity of WDR neurons is also changed in adult NP, TC animals, using plantar incision, a model of postoperative hypersensitivity (Fig. 3A).
A
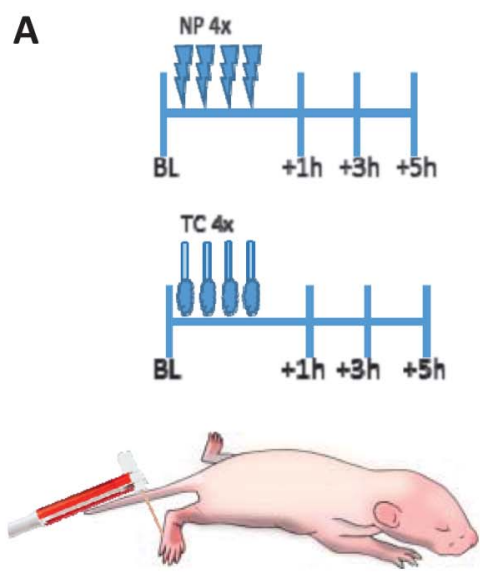

B
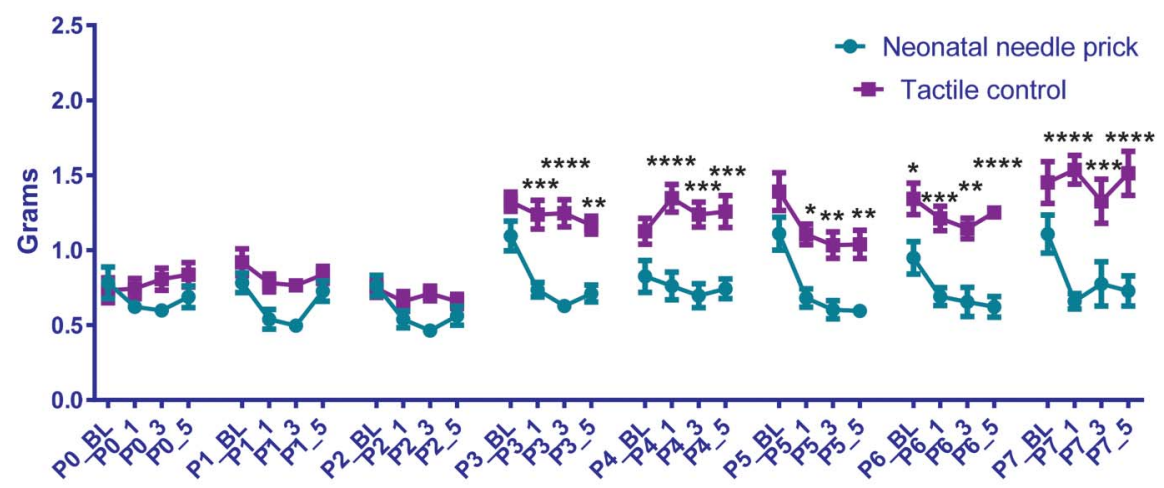

C

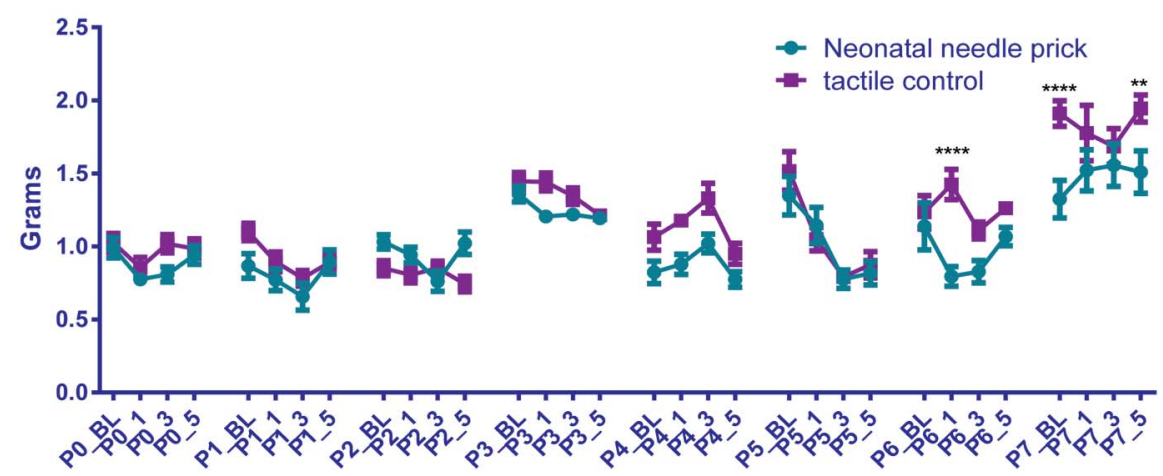

Figure 1. Mechanical sensitivity during the first week of life after repeated neonatal procedures. (A) The time course of the experiment. Rat pups (littermates) either received 4 tactile stimuli (cotton swab to the left hind paw, $N=9$ males and 2 females) or noxious NPs ( $N=10$ males and 3 females). Mechanical withdrawal thresholds in the ipsilateral and contralateral paws were tested daily using calibrated von Frey filaments, at baseline (before any repeated neonatal procedures), and at 1, 3, and 5 hours after. (B and C) Changes in mechanical withdrawal thresholds after repeated neonatal procedures in the ipsilateral (B) and contralateral (C) hind paw. Mechanical withdrawal threshold increased after the third postnatal day in tactile controls. Baseline mechanical sensitivity was comparable between the 2 neonatal procedure groups. However, after NPs, ipsilateral mechanical withdrawal thresholds were significantly lower compared with tactile controls, indicating the development of mechanical allodynia from postnatal day 3 to 7 . In addition, neonatal NPs led to significantly lower mechanical thresholds in the contralateral hind paw on postnatal day 6 and 7. BL, baseline measurement; NP, needle prick; P(0-7), postnatal day 0 to 7 ; _ 1 ,_3, and _5: measurement 1,3 , and 5 hours after $\mathrm{NP}$ /tactile stimulus; TC, tactile control stimulus. Data presented as mean $\pm \mathrm{SEM},{ }^{\star} P<0.05,{ }^{\star \star} P<0.01,{ }^{\star \star \star \star} P<0.0001$, between treatment comparisons. 


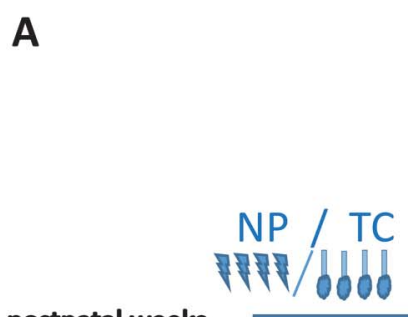

postnatal weeks

\section{1}

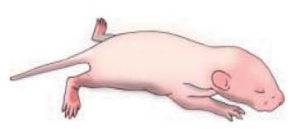

B Brush evoked response - ipsi

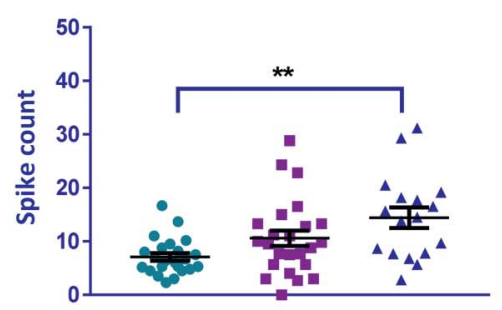

E

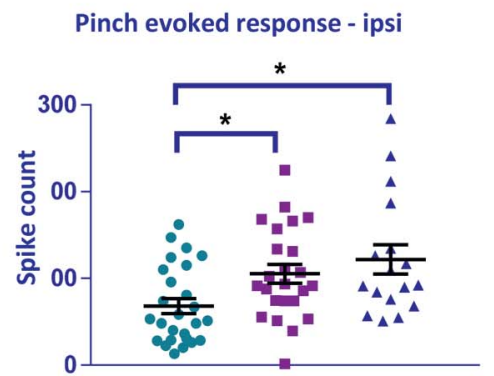

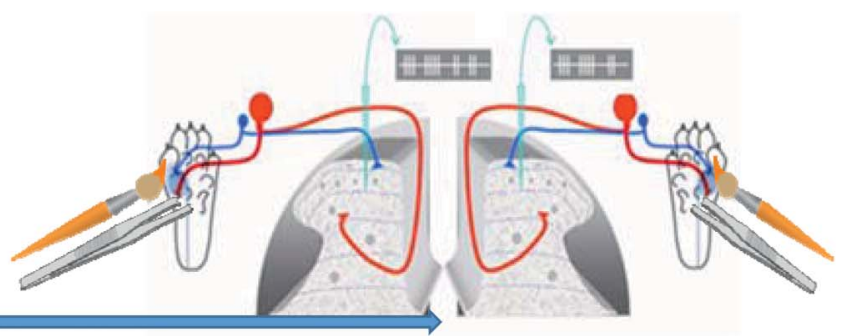

6-8

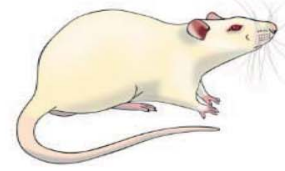

C

Brush receptive field - ipsi

D

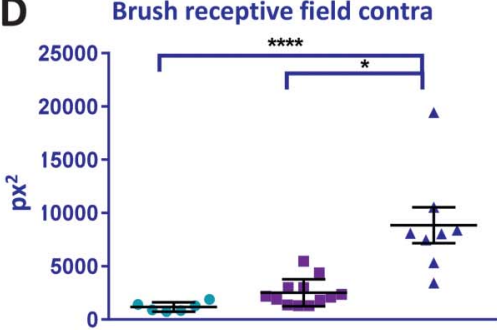

$\mathbf{F}$

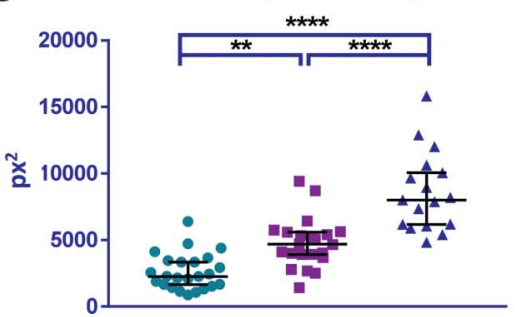

Pinch receptive field - ipsi

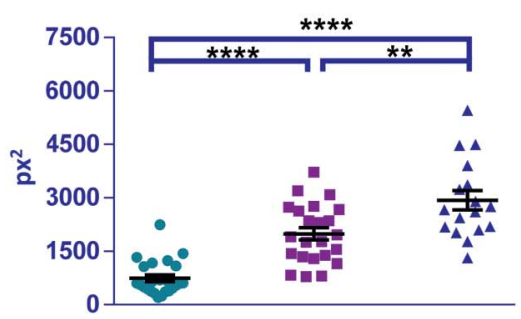

- Undisturbed control

- Neonatal tactile control

- Neonatal needle prick

Figure 2. Baseline sensitivity to cutaneous brush and noxious pinch stimulation in adult dorsal horn neurons after neonatal repeated procedures. (A) Time course of the experiment. Pups received repeated NP or TC neonatal stimuli as shown in Figure 1. A different group of pups were left undisturbed (UC). At 6 to 8 weeks of age, single-unit extracellular recordings were performed in the ipsilateral (B-E) and contralateral (F) spinal dorsal horn. Cutaneous brush- and pinch-evoked spike activity and receptive field areas were recorded in wide-dynamic-range neurons. (B) Brush-evoked spike activity was significantly greater in NP animals compared with UC. (C) Brush receptive field areas were significantly larger in NP and TC animals compared with UC, and significantly larger in NP compared with TC. (D) Pinch-evoked firing activity was significantly greater in NP compared with UC. (E) Repeated neonatal procedures significantly increased pinch receptive fields in adulthood, and this effect was greatest in NP animals. (F) Brush receptive fields were largest in the contralateral dorsal horn of NP animals. NP, needle prick; p ${ }^{2}$, pixel $^{2}$; TC, tactile control stimulus. Data presented as mean \pm SEM. ${ }^{\star} P<0.05,{ }^{\star \star} P<0.01,{ }^{\star \star \star \star} P<0.001,{ }^{\star \star \star \star} P<0.0001$, between treatment comparisons.

The mean number of spikes evoked by brush stimulation changed relative to baseline at 2 and 5 days after incision in the naive, but not NP and TC groups (Fig. 3B; effect of procedure F $(2,187)=7.07, P<0.01$; effect of day $F(2,187)=3.21, P<$ 0.05 ; UC BL: $7.1 \pm 3.4 ; 2$ days: $12.6 \pm 8.0 ; 5$ days: $12.7 \pm 6.8$; BL vs 2 days $P<0.05$; BL vs 5 days $P<0.05$, 2-way ANOVA with post hoc Bonferroni). However, significant increases in mean brush RF area were observed after incision compared with baseline (Fig. 3 C) in all treatment groups. In naive animals, brush $\mathrm{RF}$ increased after incision; this increase was stronger at 2 days compared with 5 days after incision (Fig. 3C; effect of procedure $F(2,127)=14.44, P<0.0001$; effect of day $F(2,127)=13.69$, $P<0.001 ; \mathrm{BL}: 2614 \pm 275.9 ; 2$ days: $9095 \pm 779 ; 5$ days: 5915 \pm 563 ; BL vs 2 days $P<0.0001$; BL vs 5 days $P<0.001$; 2-way ANOVA with post hoc Bonferroni). In TC animals, brush RF increased at both 2 (Fig. 3C; BL: $4767 \pm 394.7 ; 2$ days: 11,255 \pm 1054; BL vs 2 days $P<0.0001$; 2-way ANOVA with post hoc Bonferroni). Similarly, in NP animals, RF area increased at 2 and 5 days after incision (Fig. 3C; BL: $8590 \pm$ 724.2; 2 days: 11,854 \pm
976; 5 days: 11,465.85 \pm 909.91; BL vs 2 days $P<0.05$; BL vs 5 days $P<0.05$, 2-way ANOVA with post hoc Bonferroni).

Differences in postincision brush RF sizes across all treatment groups were also tested. At 2 days after incision, no significant differences were observed between naive, TC, and NP animals. At 5 days after incision, brush RF was largest in NP animals compared with both naive and TC animals (Fig. 3C; NP vs naive: $11,465.85 \pm 909.91$ vs $5915 \pm 563, P<0.0001$, NP vs TC: $11,465.85 \pm 909.91$ vs $7161.08 \pm 400.32, P<0.05$; 2-way ANOVA with post hoc Bonferroni).

Significant increases in brush-evoked activity were also observed in the contralateral dorsal horn after 5 days, where NP animals showed an increase in mean number of spikes per stimulus 2 days after incision compared with TC animals (Fig. 3D, TC vs NP $8.89 \pm 0.96$ vs $15.68 \pm 0.14, P=0.037$; 2 -way ANOVA with post hoc Bonferroni).

We next investigated the sensitivity of WDR neurons to pinch stimulation in NP, TC, and naive animals after plantar incision in the left hind paw. Neonatal repeated procedures significantly impacted 


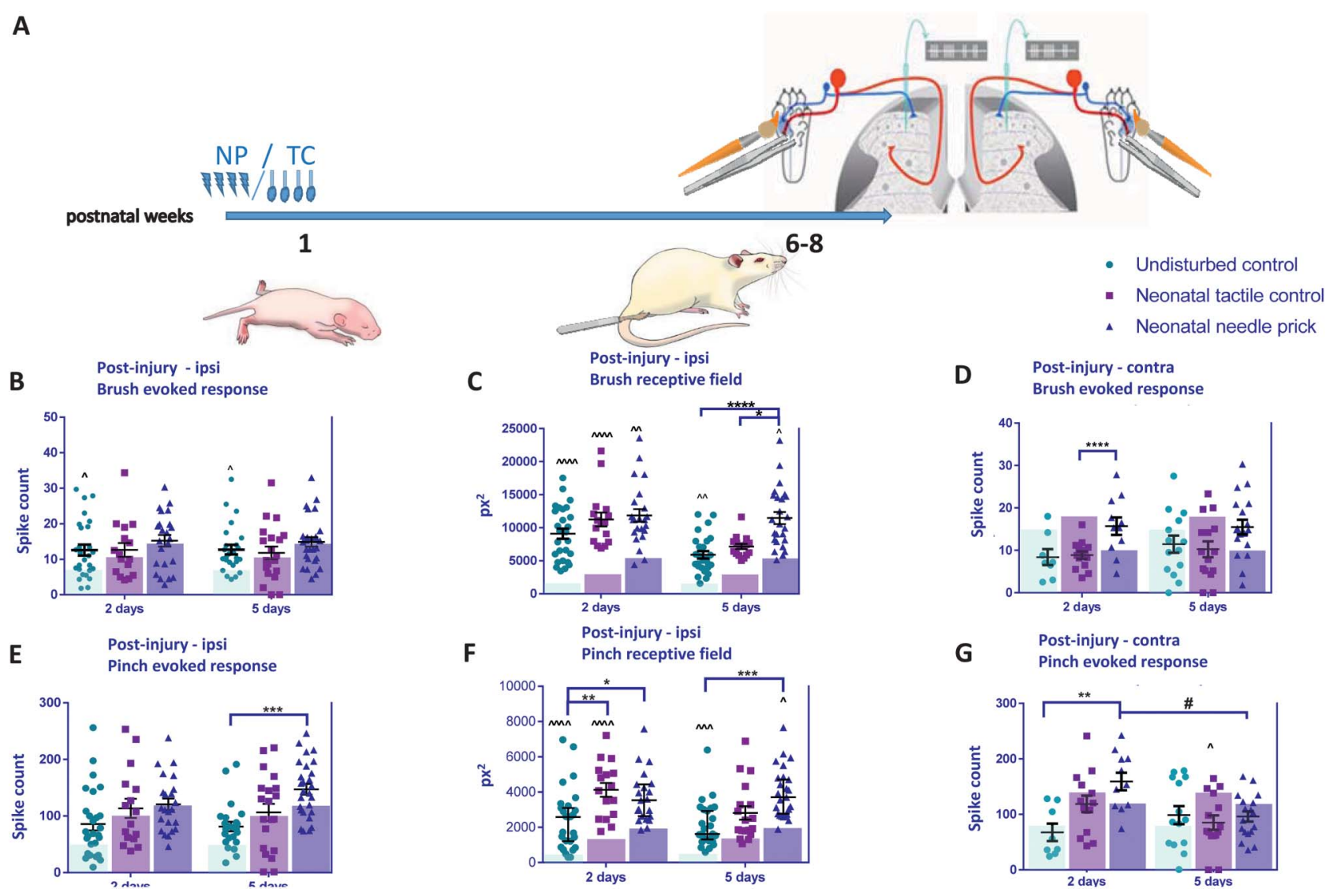

Figure 3. Postinjury sensitivity to cutaneous brush and noxious pinch stimulation in adult dorsal horn neurons after neonatal repeated procedures. (A) Time course of the experiment. Pups received neonatal stimulation or were left undisturbed. At 6 to 8 weeks of age, animals received an incision to the left hind paw on the plantar surface. Single-unit extracellular recordings were performed in the ipsilateral $(B-E)$ or contralateral $(F$ and $G)$ spinal dorsal horn 2 and 5 days later. Cutaneous brush- and pinch-evoked spike activity and receptive field areas of wide-dynamic-range neurons were recorded. (B) No changes were observed in brush responses between groups, but brush-evoked firing was significant higher in naive animals, at both 2 and 5 days after incision compared with baseline (before incision, shown as transparent coloured bars). (C) After incision, brush receptive sizes were significantly larger in all animals compared with baseline. Between-group differences were also observed. At 5 days after incision, brush receptive fields were larger in TC and NP animals compared with naive. (D) Pinchevoked firing activity after incision increased in NP animals compared with UC at 5 days after incision. (E) Pinch receptive field postincision was also significantly larger in all animals after incision compared with baseline. At 2 days, pinch receptive fields were significantly larger in TC and NP animals compared with naive. At 5 days, pinch receptive fields were larger in NP animals compared with naive. (F) Significant increases in postincision brush responses were also observed for NP animals in the contralateral dorsal horn after 2 days. (G) Contralateral pinch-evoked firing activity after incision was significantly enhanced in NP animals at day 2 postincision. NP, needle prick; $\mathrm{px}^{2}$, pixel $\mathrm{l}^{2}$; TC, tactile control stimulus; UC, undisturbed. Data presented as mean $\pm \mathrm{SEM}$, transparent coloured bars represent the baseline responses of each group. $\wedge P<0.05, \wedge \wedge P<0.01, \wedge \wedge \wedge P<0.001, \wedge \wedge \wedge \wedge P<0.0001$, within treatment comparison, compared with baseline. ${ }^{\star} P<0.05$, ${ }^{\star \star \star} P<0.001,{ }^{* \star \star \star} P<0.0001$, between treatment comparisons. $\# P<0.05$, between postincision day comparison.

on pinch-evoked activity of dorsal horn neurons after plantar incision (Fig. 3E; effect of procedure $\mathrm{F}(2,130)=10.18, P<0.0001$, 2-way ANOVA). In all 3 groups, the number of spikes evoked by noxious pinch stimulation on the RF was not significantly altered compared with baseline after incision. Pinch-evoked firing was significantly higher in NP animals 5 days after incision compared with naive animals (Fig. 3E; 5 days naive vs NP $81.38 \pm 8.54$ vs $147.2 \pm 9.5, P$ $<0.001$; 2-way ANOVA with post hoc Bonferroni).

The mean pinch RF was larger after incision compared with baseline (Fig. 3F) in all treatment groups. In naive animals, RFs were significantly larger at both 2 and 5 days after incision (BL: $742.1 \pm 97.6 ; 2$ days: $2433 \pm 342 ; 5$ days $2130 \pm 248 ; B L$ vs 2 days $P<0.0001$; BL vs 5 days $P<0.001$; 2-way ANOVA with post hoc Bonferroni). In TC animals, pinch RFs were significantly larger at 2 d (BL: $1991 \pm 170.9 ; 2$ days: $4128 \pm 397 ; P<0.0001$; 2-way ANOVA with post hoc Bonferroni) and in NP animals, at 5 days after incision (BL: $2933 \pm 267.5 ; 5$ days: $3958.78 \pm 287.55$; $P<0.05$; 2-way ANOVA with post hoc Bonferroni). We also compared pinch RF sizes between treatment groups at 2 and 5 days after incision. Needle-prick animals had enlarged pinch RFs compared with naive at 2 days (NP vs naive: $4320 \pm 521$ vs 2433 \pm 342, $P<0.05$; 2-way ANOVA with post hoc Bonferroni) and 5 days after incision (NP vs naive: $4308 \pm 445$ vs $2130 \pm 248, P<$ 0.001 ; 2-way ANOVA with post hoc Bonferroni). In addition, at 2 days after incision, RFs in TC animals were larger compared with naive (naive vs TC: $2433 \pm 342$ vs $4127.53 \pm 396.98, P=0.01$; 2-way ANOVA with post hoc Bonferroni).

In addition, contralateral pinch-evoked firing after incision was significantly enhanced in NP animals 2 days after incision compared with naive animals, and decreased to baseline values 5 days after incision (Fig. 3G, 2 days naive vs NP: $83.17 \pm 21.9$ vs $127.6 \pm$ 44.63, $P=0.01 ; 2$ days NP vs 5 days NP: $127.6 \pm 44.63$ vs $96.06 \pm$ 39.43, $P<0.05$; 2-way ANOVA with post hoc Bonferroni).

\subsection{Sensitivity to punctate mechanical stimulation is increased in adult dorsal horn neurons after early-life touch and needle prick}

Finally, we measured the sensitivity of dorsal horn WDR cells to graded mechanical punctate stimulation on the plantar skin with 
vFhs, in naive, TC, and NP animals (Fig. 4A). The average response of $3 \times 0.5$ seconds applications of each $v$ Fh was used to plot a stimulus-response curve for each neuron at baseline, 2, and 5 days after incision (Figs. 4B-G).

Baseline firing increased with vFh stimulus intensity in all groups, both ipsilateral and contralateral to treatment side (Figs. 4B-G, ipsilateral overall vFh effect: $F(6,366)=46.71, P<0.0001$; contralateral overall vFh effect: $F(6,161)=9.244, P<0.0001 ; 2$ way ANOVA with post hoc Bonferroni). However, animals that received repeated neonatal procedures (TC and NP) were more sensitive to the higher strengths of vFh when applied to the ipsilateral RF $(6-26 \mathrm{~g})$ compared with naive (Figs. 4B-C; ipsilateral overall treatment effect: $\mathrm{F}(2,61)=6.402, P<0.01$; contralateral overall treatment effect: $F(2,161)=6.081, P<0.01$; 2-way ANOVA with post hoc Bonferroni).

The postinjury sensitivity to vFhs was also tested after skin incision. After incision, vFh-evoked firing relative to baseline in naive animals increased in response to 15 and $26 \mathrm{~g}$ vFh at 2 days after incision (Figs. 4B, D, and F; VF 15 BL vs 2 days: $18.65 \pm$ 16.72 vs 30.9 $\pm 27.9, P<0.05$; VF26 BL vs 2 days: $28.14 \pm 30.8$ vs $40.99 \pm 30.5, P=0.017$; 2-way ANOVA with post hoc Bonferroni), and to 15 and $26 \mathrm{~g}$ at 5 days after incision in NP animals (VF15 BL vs 5 days $18.65 \pm 16.72$ vs $35.33 \pm 28.6, P<$ 0.01 ; VF26 BL vs 5 days: $28.14 \pm 30.8$ vs $69.75 \pm 39.6, P<$ 0.0001 ; 2-way ANOVA with post hoc Bonferroni). In addition, in $\mathrm{NP}$ animals, vFh-evoked firing in the contralateral dorsal horn in response to 8, 15, and $26 \mathrm{~g} v \mathrm{vF}$ increased 2 days after incision, and normalized compared with naive and TC animals 5 days after incision (Figs. 4C, E, and G; 2 days naive vs NP VF8: $18.21 \pm$ 21.78 vs $45.3 \pm 27.18, P=0.0036$; VF15: $22.83 \pm 20$ vs 52.82 $\pm 25.47, P=0.0010 ;$ VF26: $28.58 \pm 34.2$ vs $67.52 \pm 28.85, P<$ $0.0001 ; 2$ days TC vs NP VF8: $26 \pm 12.13$ vs $45.3 \pm 27.18, P=$ 0.0225; VF26: $48.67 \pm 20.43$ vs $67.52 \pm 28.85, P=0.027$; 2-way ANOVA with post hoc Bonferroni).

A

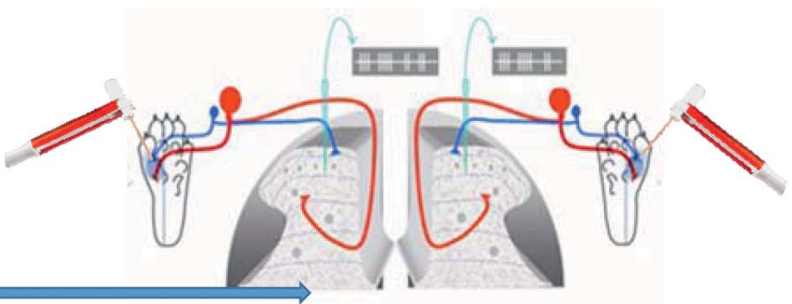

postnatal weeks

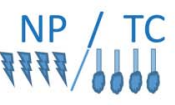

1

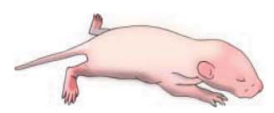

B

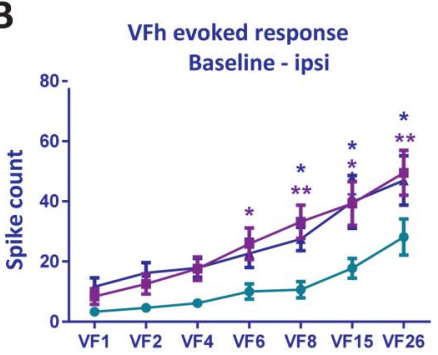

E

VFh evoked response 2 days post-incision - contra

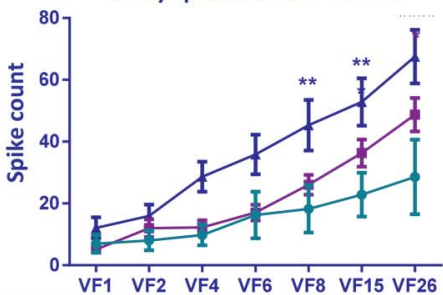

C

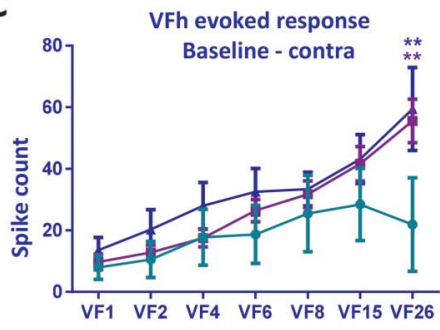

$\mathbf{F}$

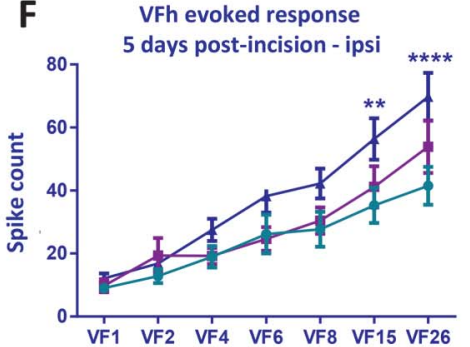
6-8

- Undisturbed control

- Neonatal tactile control

- Neonatal needle prick

Figure 4. Baseline and postinjury sensitivity to cutaneous punctate mechanical stimulation in adult dorsal horn neurons after neonatal repeated procedures. (A) Time course of the experiment. Responses in WDR neurons were recorded after graded von Frey hair (vFh) stimulation at baseline and postinjury in the ipsilateral (left panel) and contralateral (right panel) dorsal horn. Data were plotted as stimulus response curves (B) Baseline vFh sensitivity ipsilateral to injury was increased in NP animals and TC animals compared with UC. (C) Needle-prick animals displayed significantly increased vFh-evoked firing at $26 \mathrm{~g}$ in contralateral WDRs. (D) Two days after incision injury, ipsilateral stimulus response curves indicated slight hypersensitivity in NP compared with TC and UC at the highest vFh strength. (E) Two days after incision, NP animals were hypersensitive compared with TC and UC littermates in contralateral WDRs. (F) Five days after incision, NP animals were significantly more sensitive in ipsilateral WDRs than TC and UC. Changes were also observed in the contralateral dorsal horn. (G) Five days after incision, vFhevoked contralateral neuronal firing was highest in the NP group. NP, needle prick; TC, tactile control stimulus; UC, undisturbed; vFh/NF, von Frey filament; WDR, wide-dynamic range. In all figures: data presented as mean $\pm \mathrm{SEM},{ }^{*} P<0.05$, ${ }^{\star \star} P<0.01,{ }^{\star \star \star} P<0.001,{ }^{\star \star \star \star} P<0.0001$, between treatment comparisons. Asterisks are colour coded; dark blue asterisks represent between neonatal NP and undisturbed control comparisons; purple asterisks represent between neonatal tactile control and undisturbed control comparisons. 


\section{Discussion}

The aim of this study was to measure the effect of repeated neonatal sensory (painful and tactile) interventions on the sensitivity of spinal dorsal horn neurons in adulthood. We show, for the first time, that baseline innocuous and noxious mechanical sensitivity of ipsilateral spinal dorsal horn neurons is altered by both repeated tactile and NP in early life. Significant increases in neuronal sensitivity were also observed in the contralateral dorsal horn, suggesting widespread changes in spinal sensory processing. Neuronal responses to mechanical stimulation in adulthood is further enhanced in the presence of surgical skin injury. Hence, sensitivity of spinal dorsal horn neurons to a range of mechanical stimulus modalities is enhanced by repetitive neonatal sensory intervention. Although repeated noxious interventions have the greatest effects, repeated tactile interventions are also significant.

\subsection{Sensitisation of tactile and nociceptive spinal circuits as a result of abnormal sensory stimulation during the early postnatal period}

The maturation of the central nervous system is activity dependent, functional touch and pain modulation requires balance of both excitatory and inhibitory activity within the sensory network, ${ }^{4,33,34}$ and appropriate sensory signals from the periphery.

Electromyographic studies have shown that maturation of nociceptive withdrawal reflexes takes place over the first 3 postnatal weeks in the rat. ${ }^{46}$ Blockade of low-threshold afferent sensory inputs from the tail with local anaesthesia during the neonatal period inhibited refinement of tail-flick reflex, but preservation of tactile input by replacing anaesthesia with short-acting analgesic can induce adaptation in the withdrawal response. ${ }^{46}$ Other types of neonatal sensory deprivation, such as maternal separation, are routinely used to model early-life stress. ${ }^{10,50}$ Separation of male rat pups from the dam for 3 hours daily between postnatal days 2 to 14 increased visceromotor responses to phasic colonic distension at 2 months of age. ${ }^{10}$ In addition, repetitive perioperative administration of sciatic block at the time of neonatal surgery in rats inhibited the subsequent hyperalgesic responses on reinjury at the same dermatome. ${ }^{49}$ Thus, aberrant primary afferent activity may induce long-lasting hypersensitivity within central sensory circuits, which in turn alter the maturation of nociceptive processing.

Our results showed that repetitive NP in rats, a model for noxious experiences in infants admitted to neonatal intensive care unit (NICU), increased the duration of postsurgical pain sensitivity in adults. ${ }^{44}$ This is in line with other models of early-life injury which revealed long-lasting sensory behavioural changes. ${ }^{29,49}$ Importantly, in this study, we focussed on activity within the spinal dorsal horn. The spinal cord dorsal horn is the hub of pain processing and neuronal hyperexcitability within this region is a fundamental mechanism for pain sensitisation. ${ }^{4}$ By recording directly from dorsal horn neurons, rather than behavioural or reflex measures, we causally established that abnormal early-life sensory interventions directly alter baseline stimulus-evoked spike activity, and cutaneous RF areas of widedynamic neurons in the adult spinal cord. Importantly, these changes are not restricted to nociceptive, but also brush sensitivity. This highlights an advantage of in vivo dorsal horn electrophysiology where both innocuous and noxious processing can be examined in detail.

\subsection{Shaping adult pain phenotype by neonatal noxious experience}

In addition to baseline changes, we show that repetitive NPs during the first week of life in the rat strongly enhanced neuronal responses to both tactile and noxious stimulation after plantar hind paw incision (surgical pain) in the same dermatome during adulthood. ${ }^{29,49}$ Specifically, animals that received neonatal sensory interventions did not exhibit an increase in evoked firing after incision during adulthood per se, rather an expansion in RFs. This could be due to heightened basal activity of spinal neurons, which were not able to increase further in the presence of an injury. Expansion of RF areas suggests an increase in recruitment of sensory afferent terminals, perhaps by disinhibition, ${ }^{3}$ ultimately driving pain sensitization by spatial summation. ${ }^{16,52}$

Plantar hind paw incision during adulthood facilitated neuronal activity in response to mechanical stimulation in naive animals. This effect was only observed in the ipsilateral hind paw mirroring the time course of surgical pain behaviours, ${ }^{7,51}$ suggesting that a single, acute experience of surgical pain during adulthood may not be sufficient to drive global sensitization.

One limitation of this study is that it was not powered to detect sex differences. Male, but not female rats subjected to repeated needle pricking during the first week of life develop mechanical hypersensitivity to intraplantar injection of complete Freund adjuvant during adulthood. ${ }^{19}$ Similarly, intraplantar incision in P3 rats leads to mechanical, heat, and cold hypersensitivity in the ipsilateral hind paws at 4 to 6 weeks of age. ${ }^{9}$ Nonetheless, our findings and others converge onto an important conclusion: early-life sensory alterations affect maturation of sensory processing, which leads to an altered pain phenotype in adulthood. One further important point relates to the reported baseline global hyposensitivity in behavioural studies after earlylife injury. ${ }^{28,48,49}$ Here, we examined such global effects by recording from dorsal horn neurons contralateral to the treatment. We found that baseline brush activity in the contralateral dorsal horn was increased by neonatal repetitive NPs and that contralateral dorsal horn neuron brush and pinch responses were enhanced after plantar hind paw incision in adulthood. This widespread hypersensitivity agrees with previous single-unit electrophysiology studies and mapping of individual neuronal RF sizes, where neonatal surgery increased neuronal RF sizes at 6 weeks of age ${ }^{42}$ and highlights the importance of not relying reflex behaviour alone when studying sensory and pain processing.

\subsection{Early-life tactile stimulation also affects adult pain processing}

The maturation of sensory processing may be affected by several factors. In our study, we controlled for general handling or maternal separation stress by testing our NP animals alongside TC animals. Littermates were allocated to both the TC and NP group reducing any variation caused by rearing. Because neuronal hypersensitivity was persistently the strongest in NP animals, regardless of the stimulation modalities applied, pain hypersensitivity is selective to early-life pain experiences. However, the data also clearly show that repeated tactile stimulation is able to alter dorsal horn touch and pain processing. Infant rats are highly sensitive to tactile stimulation ${ }^{21}$ and this stimulation may recruit many of the same processes, to a lesser extent than noxious stimulation. In clinical studies, changes in skin conductance and behavioural arousal have been reported to be the same for noxious (heel prick) or tactile 
(routine nursery handling) stimulations, ${ }^{17}$ and a significant increase in behavioural and physiological "pain" scores has been reported on tactile stimulation compared with baseline. ${ }^{13}$ Considerable research has been undertaken in the developmental plasticity of the tactile system, ${ }^{11}$ including the effect of maternal licking and grooming during the first week of life. ${ }^{26}$ Thus, there is an increasing understanding of the dorsal horn circuitry underlying touch. ${ }^{1}$ Altogether, our findings and others underline the importance of future research in the effects of repeated handling of human neonates on their adult touch and pain processing.

\subsection{Cellular and molecular mechanisms underlying neonatal somatosensory priming of nociceptive circuits}

Neonatal injuries were shown to alter the pattern and density of afferent fibre terminations in the spinal dorsal horn. ${ }^{12,20,32}$ The retraction of low-threshold $A$ fibres from the superficial dorsal horn during the first 3 postnatal week of the rat is important for the refinement of withdrawal reflexes and maturation of sensory thresholds. ${ }^{12,30}$ If this process is disrupted, it leads to a hypersensitive phenotype in later life. ${ }^{6}$ Moreover, the higher expression of calcitonin gene-related peptide found in adult animals after neonatal repetitive procedural pain suggests an increase in nociceptive afferent sprouting. ${ }^{20}$ Importantly, early-life injuries were shown to cause substantial alterations in spike timingdependent plasticity ${ }^{23}$ and a failure of glycinergic inhibition, ${ }^{24}$ both of which enhanced dorsal horn neuron sensitivity. These neonatal interventions also occur at a period of tonic descending excitation of spinal dorsal horn neurons from brainstem control centres, ${ }^{37}$ which may be permanently altered by these events, ${ }^{47}$ for instance by increasing the opioid tone in the periaqueductal gray. $^{22}$

Substantial evidence supports a role of microglia in sensory development and plasticity. An increase in microglial reactivity accompanied enhanced pain behaviours to reinjuries in animals that had early-life noxious experiences. ${ }^{5}$ Greater phosphorylation of the signalling enzyme $\mathrm{p}-38$ mitogen-activated protein kinase (p38-MAPK) in microglia was reported in adult rats receiving plantar hind paw incision, and blockade of $\mathrm{p}-38$ MAPK reduced pain behaviours in incised adults with previous neonatal injury. ${ }^{35}$ As microglia are also important for maintenance of synaptic functions, the significance of microglia in mediating changes of neuronal outputs during early development warrants further investigation.

\subsection{Implication of findings and possible interventions}

In conclusion, our study used clinically relevant models of earlylife sensory interventions and showed that neonatal abnormal noxious and tactile stimulations persistently facilitate the activity of spinal neurons in both baseline and postsurgical conditions. Our findings and others converge onto an important conclusion: early-life sensory alterations affect maturation of sensory processing, which in turn leads to an altered somatosensory and pain phenotype in adulthood.

\section{Conflict of interest statement}

The authors have no conflict of interest to declare.

This work was supported by the Pain Knowledge Centre Maastricht and Pain Knowledge Centre Rotterdam (B.A.J., D.T., and J.P.) and the Medical Research Council UK, G0901269 (M.F.).

\section{Acknowledgements}

Author contributions: N.J. van den Hoogen, B.A. Joosten, D. Tibboel, J. Patijn, M. Fitzgerald, and C.H.T. Kwok conceived research. N. J. van den Hoogen and C.H.T. Kwok designed the research. N.J. van den Hoogen and C.H.T. Kwok performed the research. N.J. van den Hoogen, M. Fitzgerald, and C.H.T. Kwok analysed the data. M. Fitzgerald provided the resources for this research. C.H.T. Kwok and M. Fitzgerald supervised the research. N.J. van den Hoogen, M. Fitzgerald, B.A. Joosten, and C. H.T. Kwok wrote the article. All authors contributed to the article.

\section{Article history:}

Received 8 November 2017

Received in revised form 5 February 2018

Accepted 14 February 2018

Available online 16 March 2018

\section{References}

[1] Abraira VE, Kuehn ED, Chirila AM, Springel MW, Toliver AA, Zimmerman AL, Orefice LL, Boyle KA, Bai L, Song BJ, Bashista KA, O’Neill TG, Zhuo J, Tsan C, Hoynoski J, Rutlin M, Kus L, Niederkofler V, Watanabe M, Dymecki SM, Nelson SB, Heintz N, Hughes DI, Ginty DD. The cellular and synaptic architecture of the mechanosensory dorsal horn. Cell 2017;168: 295-310.e219.

[2] Anand KJ, Coskun V, Thrivikraman KV, Nemeroff CB, Plotsky PM. Longterm behavioral effects of repetitive pain in neonatal rat pups. Physiol Behav 1999;66:627-37

[3] Baccei ML, Fitzgerald M. Development of GABAergic and glycinergic transmission in the neonatal rat dorsal horn. J Neurosci 2004;24: 4749-57.

[4] Basbaum Al, Bautista DM, Scherrer G, Julius D. Cellular and molecular mechanisms of pain. Cell 2009;139:267-84.

[5] Beggs S, Currie G, Salter MW, Fitzgerald M, Walker SM. Priming of adult pain responses by neonatal pain experience: maintenance by central neuroimmune activity. Brain 2012;135:404-17.

[6] Beggs S, Torsney C, Drew LJ, Fitzgerald M. The postnatal reorganization of primary afferent input and dorsal horn cell receptive fields in the rat spinal cord is an activity-dependent process. Eur J Neurosci 2002;16: 1249-58.

[7] Brennan TJ, Vandermeulen EP, Gebhart GF. Characterization of a rat model of incisional pain. PAIN 1996;64:493-501.

[8] Brummelte S, Grunau RE, Chau V, Poskitt KJ, Brant R, Vinall J, Gover A, Synnes AR, Miller SP. Procedural pain and brain development in premature newborns. Ann Neurol 2012;71:385-96.

[9] Burke NN, Trang T. Neonatal injury results in sex-dependent nociceptive hypersensitivity and social behavioral deficits during adolescence, without altering morphine response. J Pain 2017;18:1384-96.

[10] Coutinho SV, Plotsky PM, Sablad M, Miller JC, Zhou H, Bayati Al, McRoberts JA, Mayer EA. Neonatal maternal separation alters stressinduced responses to viscerosomatic nociceptive stimuli in rat. Am J Physiol Gastrointest Liver Physiol 2002;282:G307-316.

[11] Erzurumlu RS, Gaspar P. Development and critical period plasticity of the barrel cortex. Eur J Neurosci 2012;35:1540-53.

[12] Fitzgerald M, Butcher T, Shortland P. Developmental changes in the laminar termination of a fibre cutaneous sensory afferents in the rat spinal cord dorsal horn. J Comp Neurol 1994;348:225-33.

[13] Gaspardo CM, Chimello JT, Cugler TS, Martinez FE, Linhares MB. Pain and tactile stimuli during arterial puncture in preterm neonates. PAIN 2008;140:58-64.

[14] Goldberg DS, McGee SJ. Pain as a global public health priority. BMC Public Health 2011;11:770.

[15] Grunau RE, Whitfield MF, Petrie-Thomas J, Synnes AR, Cepeda IL, Keidar A, Rogers M, Mackay M, Hubber-Richard P, Johannesen D. Neonatal pain, parenting stress and interaction, in relation to cognitive and motor development at 8 and 18 months in preterm infants. PAIN 2009;143:138-46.

[16] Hamalainen MM, Gebhart GF, Brennan TJ. Acute effect of an incision on mechanosensitive afferents in the plantar rat hindpaw. J Neurophysiol 2002;87:712-20.

[17] Hellerud BC, Storm H. Skin conductance and behaviour during sensory stimulation of preterm and term infants. Early Hum Dev 2002;70:35-46. 
[18] Howard RF, Walker SM, Mota PM, Fitzgerald M. The ontogeny of neuropathic pain: postnatal onset of mechanical allodynia in rat spared nerve injury (SNI) and chronic constriction injury (CCI) models. PAIN 2005; 115:382-9.

[19] Knaepen L, Patijn J, Tibboel D, Joosten EA. Sex differences in inflammatory mechanical hypersensitivity in later life of rats exposed to repetitive needle pricking as neonates. Neurosci Lett 2012;516:285-9.

[20] Knaepen L, Patijn J, van Kleef M, Mulder M, Tibboel D, Joosten EA. Neonatal repetitive needle pricking: plasticity of the spinal nociceptive circuit and extended postoperative pain in later life. Developmental Neurobiol 2013;73:85-97.

[21] Koch SC, Fitzgerald M. Activity-dependent development of tactile and nociceptive spinal cord circuits. Ann N Y Acad Sci 2013;1279:97-102.

[22] Laprairie JL, Murphy AZ. Neonatal injury alters adult pain sensitivity by increasing opioid tone in the periaqueductal gray. Front Behav Neurosci 2009;3:31

[23] Li J, Baccei ML. Neonatal tissue damage promotes spike timingdependent synaptic long-term potentiation in adult spinal projection neurons. J Neurosci 2016;36:5405-16.

[24] Li J, Blankenship ML, Baccei ML. Deficits in glycinergic inhibition within adult spinal nociceptive circuits after neonatal tissue damage. PAIN 2013; 154:1129-39.

[25] Marlow N, Rose AS, Rands CE, Draper ES. Neuropsychological and educational problems at school age associated with neonatal encephalopathy. Arch Dis Child Fetal Neonatal Ed 2005;90:F380-387.

[26] Meaney MJ, Szyf M. Environmental programming of stress responses through DNA methylation: life at the interface between a dynamic environment and a fixed genome. Dialogues Clin Neurosci 2005;7: 103-23.

[27] Peters JW, Koot HM, de Boer JB, Passchier J, Bueno-de-Mesquita JM, de Jong FH, Duivenvoorden HJ, Tibboel D. Major surgery within the first 3 months of life and subsequent biobehavioral pain responses to immunization at later age: a case comparison study. Pediatrics 2003: 111:129-35.

[28] Ren K, Anseloni V, Zou SP, Wade EB, Novikova SI, Ennis M, Traub RJ, Gold MS, Dubner R, Lidow MS. Characterization of basal and reinflammation-associated long-term alteration in pain responsivity following short-lasting neonatal local inflammatory insult. PAIN 2004; 110:588-96.

[29] Ren K, Novikova SI, He F, Dubner R, Lidow MS. Neonatal local noxious insult affects gene expression in the spinal dorsal horn of adult rats. Mol Pain 2005;1:27.

[30] Ririe DG, Vernon TL, Tobin JR, Eisenach JC. Age-dependent responses to thermal hyperalgesia and mechanical allodynia in a rat model of acute postoperative pain. Anesthesiology 2003;99:443-8.

[31] Roofthooft DW, Simons SH, Anand KJ, Tibboel D, van Dijk M. Eight years later, are we still hurting newborn infants? Neonatology 2014;105: 218-26.

[32] Ruda MA, Ling QD, Hohmann AG, Peng YB, Tachibana T. Altered nociceptive neuronal circuits after neonatal peripheral inflammation. Science 2000;289:628-31.

[33] Sandkuhler J. The organization and function of endogenous antinociceptive systems. Prog Neurobiol 1996:50:49-81.

[34] Sandkuhler J. Models and mechanisms of hyperalgesia and allodynia. Physiol Rev 2009;89:707-58.
[35] Schwaller F, Beggs S, Walker SM. Targeting p38 mitogen-activated protein kinase to reduce the impact of neonatal microglial priming on incision-induced hyperalgesia in the adult rat. Anesthesiology 2015;122: 1377-90.

[36] Schwaller F, Fitzgerald M. The consequences of pain in early life: injuryinduced plasticity in developing pain pathways. Eur J Neurosci 2014;39: 344-52.

[37] Schwaller F, Kwok C, Fitzgerald M. Postnatal maturation of the spinalbulbo-spinal loop: brainstem control of spinal nociception is independent of sensory input in neonatal rats. PAIN 2016;157:677-86.

[38] Slater R, Fabrizi L, Worley A, Meek J, Boyd S, Fitzgerald M. Premature infants display increased noxious-evoked neuronal activity in the brain compared to healthy age-matched term-born infants. Neuroimage 2010; 52:583-9.

[39] Sternberg WF, Scorr L, Smith LD, Ridgway CG, Stout M. Long-term effects of neonatal surgery on adulthood pain behavior. PAIN 2005;113: 347-53.

[40] Taddio A, Shah V, Gilbert-MacLeod C, Katz J. Conditioning and hyperalgesia in newborns exposed to repeated heel lances. JAMA 2002;288:857-61.

[41] Tich SN, Anderson PJ, Hunt RW, Lee KJ, Doyle LW, Inder TE. Neurodevelopmental and perinatal correlates of simple brain metrics in very preterm infants. Arch Pediatr Adolesc Med 2011;165:216-22.

[42] Torsney C, Fitzgerald M. Spinal dorsal horn cell receptive field size is increased in adult rats following neonatal hindpaw skin injury. J Physiol 2003;550:255-61.

[43] Van den Hoogen NJ, Patijn J, Tibboel D, Joosten EA. Neonatal plasticity of the nociceptive system: mechanisms, effects, and treatment of repetitive painful procedures during NICU admittance. Curr Pharm Des 2017;23:5902-10.

[44] van den Hoogen NJ, Tibboel D, Honig WM, Hermes D, Patijn J, Joosten EA. Neonatal paracetamol treatment reduces long-term nociceptive behaviour after neonatal procedural pain in rats. Eur J Pain 2016;20: 1309-18.

[45] Vardeh D, Mannion RJ, Woolf CJ. Toward a mechanism-based approach to pain diagnosis. J Pain 2016;17:T50-69.

[46] Waldenstrom A, Thelin J, Thimansson E, Levinsson A, Schouenborg J. Developmental learning in a pain-related system: evidence for a crossmodality mechanism. J Neurosci 2003;23:7719-25.

[47] Walker SM, Fitzgerald M, Hathway GJ. Surgical injury in the neonatal rat alters the adult pattern of descending modulation from the rostroventral medulla. Anesthesiology 2015;122:1391-400.

[48] Walker SM, Franck LS, Fitzgerald M, Myles J, Stocks J, Marlow N. Longterm impact of neonatal intensive care and surgery on somatosensory perception in children born extremely preterm. PAIN 2009;141:79-87.

[49] Walker SM, Tochiki KK, Fitzgerald M. Hindpaw incision in early life increases the hyperalgesic response to repeat surgical injury: critical period and dependence on initial afferent activity. PAIN 2009;147:99-106.

[50] Weaver SA, Diorio J, Meaney MJ. Maternal separation leads to persistent reductions in pain sensitivity in female rats. J Pain 2007;8:962-9.

[51] Xu J, Brennan TJ. Comparison of skin incision vs. skin plus deep tissue incision on ongoing pain and spontaneous activity in dorsal horn neurons. PAIN 2009;144:329-39.

[52] Zahn PK, Brennan TJ. Incision-induced changes in receptive field properties of rat dorsal horn neurons. Anesthesiology 1999;91:772-85. 